\title{
Immune Dysregulation Resulting From Impaired Interleukin-10 And The Interleukin-10 Receptor Signaling: A Systematic Review of 284 Monogenic Patients
}

\section{Niusha Sharifinejad}

Alborz University of Medical Sciences

\section{Majid Zaki-Dizaji}

Baqiyatallah University of Medical Sciences

Roya Sepahvandi

Alborz University of Medical Sciences

\section{Farimah Fayyaz}

Alborz University of Medical Sciences

Maria Marluce dos Santos Vilela

State University of Campinas (UNICAMP). Campinas

\section{Gehad ElGhazali}

Sheikh Khalifa Medical City

\section{Hassan Abolhassani}

Tehran University of Medical Sciences

\section{Hans D Ochs}

Seattle Children's Research Institute

Gholamreza Azizi ( $\nabla$ azizi@abzums.ac.ir)

ABZUMS https://orcid.org/0000-0001-5658-2511

\section{Research Article}

Keywords: IL-10 receptor deficiency, IL-10 deficiency, Very early-onset IBD, Inborn errors of immunity, Primary immunodeficiency

Posted Date: December 15th, 2021

DOI: https://doi.org/10.21203/rs.3.rs-1160985/v1

License: (9) (1) This work is licensed under a Creative Commons Attribution 4.0 International License. Read Full License 
Version of Record: A version of this preprint was published at Clinical \& Experimental Immunology on April 27th, 2022. See the published version at https://doi.org/10.1093/cei/uxac040. 


\section{Abstract}

Purpose: Interleukin-10 (IL-10) and IL-10 receptor (IL-10R) deficiency are monogenic inborn errors of immunity (IEI) causing early-onset inflammatory bowel diseases (IBD).

Methods: We systematically reviewed articles that included related keywords using PubMed, Web of Science, and Scopus databases. The articles were screened for eligibility criteria before data extraction.

Results: We assessed 284 patients (44.1\% female) with IL-10 and/or IL-10R deficiencies who were predominantly from China (41.0\%), Italy (14.1\%), and South Korea (8.6\%). The median age of onset was 1.0 (0.3-4.0) months with a median age of genetic diagnosis at 16.0 (7.4-81.0) months. Consanguinity was reported in all evaluable patients with IL-10 deficiency and in $38.8 \%$ of patients with IL-10R deficiency (23.4\% of patients with IL-10RA, and 79.4\% of patients with IL-10RB deficiency). The most prevalent mutations in $I L-10 R A$ were c.301C $>T$ (p.R101W) and c.537G $>A$ (p.T179T), those in $I L-10 R B$ were c.139A $>$ G (p.K47E) and c.611G>A (p.W204X). Autoimmunity and enteropathy were present in all cases. The first presentation of both groups was protracted diarrhea (45.7\%), bloody diarrhea (18.1\%), and colitis (15.7\%). Patients with IL-10R deficiency had a high frequency of dermatologic manifestations (50.5\%) and failure to thrive $(60 \%)$, while IL-10 deficient patients lacked those complications. In the majority of patients, the basic immunologic parameters were in normal ranges. Of the entire publications, $30 \%$ underwent hemopoietic stem cell transplantation, $61.5 \%$ surgery, and $87 \%$ immunosuppressive treatment. The ten-year survival rate was higher in patients with IL-10 deficiency than in patients with IL-10R deficiency.

Conclusion: IL-10/IL-10R deficiency predominantly presents with treatment-resistant, early-onset IBD within the first months of life. No clear genotype-phenotype correlation was present among these patients. The high prevalence of distinct clinical manifestations reported in IL-10RA- and IL-10RBdeficient patients might be attributable to the interaction between the target tissue and cytokines other than IL- 10 capable of binding to IL-10RB. These results gain translational significance by contributing to earlier diagnosis, adequate therapy, and avoiding delay in the diagnosis and unfavorable outcome.

\section{Introduction}

The Interleukin-10 (IL-10) protein encoded by the IL-10 gene is a major immune-regulatory cytokine produced primarily by monocytes, macrophage, dendritic cells (DCs), and lymphocytes with profound anti-inflammatory functions (1). IL-10 binds to its heterotetrameric receptor, IL-10 receptor (IL-10R) A and B, leading to Janus kinase 1 (JAK 1) and Tyrosine Kinase 2 (TYK2)-mediated phosphorylation of phospho-signal transducer and activator of transcription 3 (STAT3). In turn, STAT3 translocates to the nucleus where it drives the expression of anti-inflammatory mediators. STAT3 down-regulates the expression of T-helper (Th1) cytokines and antigen presentation by reducing the expression of major histocompatibility complex (MHC)-class II and co-stimulatory molecules on macrophages, thereby inhibiting $T$ cell activation. It also controls macrophage activation by reprogramming essential metabolic 
pathways (2). In addition, IL-10 enhances B cell survival and proliferation, regulates antibody production, can inhibit nuclear factor kappa B (NF-KB) activity, and is involved in the regulation of the JAK-STAT signaling pathway (2). On the other hand, the IL-10RB subunit is not only activated by IL-10, but also by IL-22, IL-26, and IL-28A,B/IL29, respectively (3). The IL 10 gene is located on chromosome 1q32.1 consisting of 5 exons and 178 amino acids (NP_000563.1). The IL-10RA and IL-10RB receptors genes are located on chromosomes 11q23.3 and 21q22.11 both consisting of 7 exons and 578 and 325 amino acids, respectively (NP_001549.2, NP_000619.3). Importantly, IL-10/IL-10R signaling defects lead to a hyper-inflammatory state, predominantly in the intestinal tissue, especially within the first months of life $(4,5)$.

IL-10 (MIM: 614395) and IL-10R (MIM: 613148 and 612567) deficiencies have been recognized by the international union of immunological societies (IUIS) Classification Committee for inborn errors of immunity (IEI) as "diseases of immune dysregulation with colitis" and more recently as one of the "primary immune regulatory disorders" which includes more than 129 genes (6). Pediatric inflammatory bowel disease (IBD) is classified into five major groups according to the age at onset: pediatric-onset IBD (younger than 17 years), early-onset IBD (younger than 10 years), very early onset IBD (younger than 6 years), infantile (and toddler) onset IBD (younger than 2 years old), and neonatal IBD (within the first 28 days of age) (7). Thus, IL-10/IL-10R deficiencies are mainly categorized as infantile-onset and very early onset IBD or VEOIBD (8). On the other hand, undetermined IBD (UIBD) is a rare subgroup of pediatric IBD allocated to cases whom endoscopic and histological findings are not adequate to differentiate between ulcerative colitis and Crohn's disease (9).

Even though IBD is the most important clinical presentation among IL-10/IL-10R-deficient patients, other extra-intestinal symptoms include folliculitis, arthritis, and respiratory diseases (10). An increased susceptibility for B cell lymphoma has also been reported in patients with IL-10/IL-10R deficiency (11). Except for slight alterations in immunoglobulins levels and STAT3 or IL-10 expression detected in some cases $(12,13)$, inflammatory markers and immunological evaluations are generally normal (13). Similar to a subset of other monogenic IEl, allogeneic hemopoietic stem cell transplantation (HSCT) is currently the standard curative approach in IL-10/IL-10R deficiencies which are in most cases resistant to standard immunosuppressive therapy (14). A few studies suggested that IL-10/IL-10R-deficient patients might benefit from recombinant human IL-10 or IL-1-targeting therapies $(15,16)$.

Given the different therapeutic approaches reported for IL-10/IL-10R-deficient patients in comparison to other IBD-affected patients and the necessity of early diagnosis and intervention in these patients, we systematically reviewed the clinical, immunological, and genetic features as well as the therapeutic options used for patients with IL-10/IL-10R deficiencies to attain a comprehensive view of this disease, assist physicians in establishing an earlier diagnosis and improve the patients' prognosis.

\section{Methods}

\subsection{Search Strategy}


A comprehensive search up to the 29th of April 2021, and limited to articles written in English, was performed using PubMed, Web of Science, and Scopus databases. The used keywords were "interleukin 10 deficiency”, “IL-10 deficiency”, “IL10 mutation”, "interleukin 10 receptor deficiency”, "IL-10R deficiency”, "IL10R mutation”, "IL-10RA mutation”, “IL-10RB mutation”, "VEO-IBD”, “EO-IBD”, "very early onset inflammatory bowel disease", "early onset IBD", or "infantile inflammatory bowel disease". The search was conducted using these terms in the keywords, titles, and abstracts of articles. The reference lists of all full-text articles and major reviews were manually searched for additional studies.

\subsection{Study Selection}

The articles were first screened based on the title and abstract to exclude all irrelevant studies and were classified into three groups (include, exclude, or unclear); the full-text version of all "unclear" articles were checked and categorized into one of the two other categories (include or exclude). Similar to our previous article (17), the full-text of each included paper was assessed for eligibility criteria, requiring reporting at least one patient with a genetic diagnosis of IL-10/IL-10R deficiency. When necessary, the corresponding authors of the selected articles were contacted.

\subsection{Data Extraction}

Two authors independently extracted the data from the included studies. The following data were collected from each article: publication year, the number of patients, demographic details, and clinical, laboratory, and molecular data of each patient with IL-10 or IL-10R deficiency. The immunologic data quality was evaluated based on normal ranges available in each article or age-matched normal control ranges when the normal ranges were not mentioned. Duplicated cases were identified and removed. The medical records of all papers were gathered and subsequently merged. A third author was consulted in case of disagreements between the two reviewers.

\subsection{Statistical Analysis}

All statistical analyses were performed using the SPSS software (v. 26.0, Chicago, IL) and GraphPad Prism (V. 8.0, San Diego, CA). Central and descriptive statistics were presented for quantitative data. Median and interquartile ranges (IQR) were calculated for quantitative variables with the abnormal distribution. Qualitative data were interpreted using absolute numbers and percentages. To achieve a better understanding of IL-10/IL-10R deficiencies, we compared the patients within two groups: IL-10 deficiency vs. IL-10R deficiency and IL-10RA deficiency vs. IL-10RB deficiency. Independent T-test, Wilcoxon, Chi-square, or Fisher exact tests were utilized to compare the groups. Kaplan-Meier curve and log-rank test were used to compare different survival estimates. All statistical tests were two-tailed. A $P$ value $<0.05$ was considered statistically significant.

\section{Results}

\subsection{Study Characteristics}


The literature search yielded a total of 1964 articles. After removing the duplicated articles, 1706 articles were screened according to title and abstract, resulting in the exclusion of 1600 articles. Three additional articles were identified during the hand-search of full-text articles and major reviews. As shown in Figure 1,109 articles fulfilled the inclusion criteria and were subsequently assessed in the full-text. Of those 109 articles, 62 with a total of 306 patients were included in the systematic review. After the removal of duplicate cases, 284 unique patients remained for data analysis.

\subsection{Epidemiologic characteristics}

A total of 284 patients (116 female, 147 male, and 21 with unknown gender) with IL-10 and/or IL-10R deficiency were assessed. Among them, 18 (6.3\%) were IL-10 deficient and 266 (93.7\%) were IL-10R deficient [IL-10RA: 188, IL-10RB: 69, combined heterozygous IL-10RA/RB: 7, and for two patients the IL$10 \mathrm{R}$ variant was not specified but was confirmed using proteomics evidence]. The patients originated predominantly from China $41.0 \%$ (105 of 256), Italy $14.1 \%$ (36 of 256), and South Korea 8.6\% (22 of 256); the nationality of 28 patients were not mentioned. A total of 174 patients $(68.0 \%)$ originated from Asia, 62 from Europe (24.2\%), and 20 from America (7.8\%, Figure 2). The median (IQR) age of onset of patients' symptoms was 1.0 (0.3-4.0) month with a median (IQR) diagnostic delay of 12.0 (6.0-71.7) months. Forty-one percent of cases (55 of 150 patients with available data) were born to consanguineous parents and $28.2 \%$ (42 of 149) had a positive family history of IEl. Most of those (38 cases) had a history of familial early-onset IBD. About nineteen percent of the patients (41 of 214 with available life/death state) died at a median (IQR) age of 1.4 (0.9-3.9) years during an average of 2.6 (1.2-8.0) years of follow-up. The most common causes of death were infections (19 cases), lymphoproliferative disease (2 cases), organ failure ( 2 cases), and the result of severe infantile IBD ( 2 cases). The demographic data of all patients are summarized in Table 1. Among patients with IL-10, IL-10RA, and IL-10RB deficiencies, the male to female ratio was $4,1.3$, and 1.03 , respectively. Consanguinity was reported in all evaluable patients with IL-10 deficiency whereas, only $38.8 \%$ of patients with IL-10R deficiency had consanguineous parents $(p=0.010)$. The frequency of consanguineous marriages was also lower in patients with IL-10RA deficiency than patients with IL-10RB deficiency $(23.4 \%$ vs. $79.4 \%, \mathrm{p}<0.001)$. Age at death was significantly lower in patients with IL-10RA deficiency than IL-10RB deficiency [1.2 (0.8-2.1) vs. 4.5 (1.49.0) years, $p=0.014]$, while none of the IL-10-deficient patients had deceased. 
Table 1

Demographic data of patients with IL-10/IL-10R deficiencies.

\begin{tabular}{|c|c|c|c|c|c|c|c|}
\hline Parameters & $\begin{array}{l}\text { Total } \\
(n=284)\end{array}$ & $\begin{array}{l}\text { IL-10 } \\
(n=18)\end{array}$ & $\begin{array}{l}\text { IL-10R } \\
(n=266)\end{array}$ & $\begin{array}{l}P- \\
\text { value }\end{array}$ & $\begin{array}{l}\text { IL-10RA } \\
(n=188)\end{array}$ & $\begin{array}{l}\text { IL- } \\
\text { 10RB } \\
(n=69)\end{array}$ & $\begin{array}{l}P- \\
\text { value }\end{array}$ \\
\hline Sex ratio, $M / F,(n=263)$ & $147 / 116$ & $8 / 2$ & $139 / 114$ & 0.193 & $104 / 80$ & $31 / 30$ & 0.438 \\
\hline $\begin{array}{l}\text { Family history of IEI, } n \text {, } \\
(\%)(n=149)\end{array}$ & $\begin{array}{l}42 \\
(28.2)\end{array}$ & 0 & $42(29)$ & 0.577 & $\begin{array}{l}34 \\
(27.9)\end{array}$ & $\begin{array}{l}7 \\
(33.3)\end{array}$ & 0.609 \\
\hline $\begin{array}{l}\text { Consanguinity, } n,(\%) \\
(n=134)\end{array}$ & $\begin{array}{l}55 \\
(41.0)\end{array}$ & $5(100)$ & $\begin{array}{l}50 \\
(38.8)\end{array}$ & 0.010 & $\begin{array}{l}22 \\
(23.4)\end{array}$ & $\begin{array}{l}27 \\
(79.4)\end{array}$ & $<0.001$ \\
\hline Dead, $n,(\%)(n=214)$ & $\begin{array}{l}41 \\
(19.2)\end{array}$ & 0 & $\begin{array}{l}41 \\
(19.6)\end{array}$ & 0.586 & $\begin{array}{l}32 \\
(20.8)\end{array}$ & $\begin{array}{l}9 \\
(17.3)\end{array}$ & 0.588 \\
\hline $\begin{array}{l}\text { Age at onset, } m \text {, median } \\
(\text { (IQR), }(n=263)\end{array}$ & $\begin{array}{l}1.0(0.3- \\
4.0)\end{array}$ & $\begin{array}{l}2.0 \\
(1.0- \\
4.0)\end{array}$ & $\begin{array}{l}1.0(0.3- \\
4.0)\end{array}$ & 0.444 & $\begin{array}{l}0.9 \\
(0.2- \\
3.0)\end{array}$ & $\begin{array}{l}2.6 \\
(1.0- \\
4.0)\end{array}$ & 0.309 \\
\hline $\begin{array}{l}\text { Age at molecular } \\
\text { diagnosis, m, median } \\
\text { (IQR), }(n=66)\end{array}$ & $\begin{array}{l}16.0 \\
(7.4- \\
81.0)\end{array}$ & $\begin{array}{l}41.0 \\
(6.0- \\
116.5)\end{array}$ & $\begin{array}{l}14.0 \\
(7.7- \\
76.5)\end{array}$ & 0.765 & $\begin{array}{l}14.0 \\
(8.2- \\
91.5)\end{array}$ & $\begin{array}{l}17.0 \\
(6.0- \\
72.0)\end{array}$ & 0.676 \\
\hline $\begin{array}{l}\text { Delay in diagnosis, m, } \\
\text { median (IQR), }(n=41)\end{array}$ & $\begin{array}{l}12.0 \\
(6.0- \\
71.7)\end{array}$ & $\begin{array}{l}30.4 \\
(2.5- \\
119.9)\end{array}$ & $\begin{array}{l}12.0 \\
(6.2- \\
71.7)\end{array}$ & 0.924 & $\begin{array}{l}12.0 \\
(7.0- \\
72.0)\end{array}$ & $\begin{array}{l}16.3 \\
(3.5- \\
75.1)\end{array}$ & 0.750 \\
\hline $\begin{array}{l}\text { Follow up, y, median } \\
\text { (IQR), ( }(n=157)\end{array}$ & $\begin{array}{l}2.6(1.2- \\
8.0)\end{array}$ & $\begin{array}{l}7.0 \\
(3.6- \\
9.4)\end{array}$ & $\begin{array}{l}2.5(1.2- \\
8.0)\end{array}$ & 0.805 & $\begin{array}{l}2.0 \\
(1.1- \\
6.1)\end{array}$ & $\begin{array}{l}4.7 \\
(2.5- \\
13.0)\end{array}$ & 0.022 \\
\hline $\begin{array}{l}\text { Age at death, } y \text {, median } \\
(\text { (IQR), }(n=28)\end{array}$ & $\begin{array}{l}1.4(0.9- \\
3.9)\end{array}$ & - & $\begin{array}{l}1.4(0.9- \\
3.9)\end{array}$ & - & $\begin{array}{l}1.2 \\
(0.8- \\
2.1)\end{array}$ & $\begin{array}{l}4.5 \\
(1.4- \\
9.0)\end{array}$ & 0.014 \\
\hline \multicolumn{8}{|c|}{ M; Male, F; Female, IEl; Inborn error of immunity, y; year, m; month. } \\
\hline \multicolumn{8}{|c|}{ The median is shown [with 25th and 75th percentiles]. } \\
\hline \multicolumn{8}{|c|}{ * $p$-value is statistically significant $<0.05$} \\
\hline
\end{tabular}

\subsection{Molecular findings}

Of the 284 reported patients representing 279 families, detailed genetic data were not available from 19 (7\%) patients. Most of the cases with determined genetic defects carried mutations in the IL-10-RA gene $(69 \%)$. Among the 265 patients (263 families) with a confirmed mutation, 8 patients have mutations in IL10,183 and 68 patients have mutations in IL-10RA and IL10-RB respectively, and 6 patients with both IL10-RA and RB mutations. Table 2 provides detailed information on mutation confirmed cases from individual families. 
Table 2

Families with reported mutations in IL-10, IL-10RA and IL-10RB genes.

\begin{tabular}{|c|c|c|c|c|c|}
\hline Gene & $\begin{array}{l}\text { Families } \\
(n=263)\end{array}$ & Zygosity & $\begin{array}{l}\text { Different } \\
\text { mutation }\end{array}$ & Frequent Mutations & Mutation type \\
\hline \multirow[t]{2}{*}{ IL-10 } & \multirow[t]{2}{*}{8} & \multirow[t]{2}{*}{ Hom:8 } & \multirow[t]{2}{*}{2} & c. $337 \mathrm{G}>\mathrm{A}$ & \multirow[t]{2}{*}{ Missense } \\
\hline & & & & c. $458 \mathrm{G}>\mathrm{A}$ & \\
\hline \multirow[t]{5}{*}{ IL-10RA } & \multirow[t]{5}{*}{181} & Hom:71 & \multirow[t]{5}{*}{58} & c. $301 \mathrm{C}>\mathrm{T}(26 \%)$ & Missense (63\%) \\
\hline & & Het $^{*}: 5$ & & c. $537 \mathrm{G}>\mathrm{A}(21 \%)$ & \multirow{2}{*}{$\begin{array}{l}\text { Silent/splicing } \\
(22 \%)\end{array}$} \\
\hline & & Com.Het:99 & & c. $1051 A>G(4 \%)$ & \\
\hline & & \multirow[t]{2}{*}{$>2$ mut $^{* \star}: 6$} & & \multirow[t]{2}{*}{ c. $299 \mathrm{~T}>\mathrm{G}(4 \%)$} & $\begin{array}{l}\text { Splice site }(3 \%) \\
\text { Large InDel (4\%) }\end{array}$ \\
\hline & & & & & $\begin{array}{l}\text { InDel-Frameshift } \\
(2 \%)\end{array}$ \\
\hline \multirow[t]{6}{*}{ IL-10RB } & \multirow[t]{6}{*}{68} & Hom:59 & \multirow[t]{6}{*}{30} & \multirow{2}{*}{$\begin{array}{l}\text { c. } 139 A>G(16 \%) \\
\text { c. } 477 G>A(10 \%)\end{array}$} & Missense (31\%) \\
\hline & & \multirow[t]{5}{*}{ Com.Het: 9} & & & Nonsense (29\%) \\
\hline & & & & C.011 & Splice site (11\%) \\
\hline & & & & $\begin{array}{l}\text { C. } \\
(5 \%)\end{array}$ & $\begin{array}{l}\text { InDel-Frameshift } \\
(10 \%)\end{array}$ \\
\hline & & & & & Large InDel (13\%) \\
\hline & & & & & $\begin{array}{l}\text { 3'UTR, promoter } \\
(5 \%)\end{array}$ \\
\hline \multirow{5}{*}{$\begin{array}{l}\text { IL-10RA/ } \\
\text { IL-10RB }\end{array}$} & \multirow[t]{5}{*}{6} & \multirow{5}{*}{$\begin{array}{l}\text { Double } \\
\text { Het }^{\#:} 6\end{array}$} & \multirow[t]{5}{*}{3} & IL-10RA: & \multirow[t]{5}{*}{ Missense } \\
\hline & & & & c. $475 A>G$ & \\
\hline & & & & c. $1051 A>G$ & \\
\hline & & & & IL-10RB: & \\
\hline & & & & c. $139 A>G$ & \\
\hline \multicolumn{6}{|c|}{$\begin{array}{l}\text { Hom: homozygote, Het: heterozygote, Com. Het: compound heterozygote, InDel: both insertion or } \\
\text { deletion, insertion and deletion } \\
\text { *In these cases, IL-10RA mutations were in a heterozygous state and no additional homozygote or } \\
\text { compound heterozygote mutations were identified. }\end{array}$} \\
\hline \multicolumn{6}{|c|}{ **In these cases, more than 2 variants were reported. } \\
\hline \multicolumn{6}{|c|}{ \#These cases have heterozygous mutations in both IL-10RA/ and IL-10RB. } \\
\hline
\end{tabular}

A total of 90 unique mutations were detected (Table 2, Figure 3). The most prevalent mutations among patients with IL-10RA were c.301C>T (exon 3, p.R101W) and c.537G >A (exon 4, p.T179T-splicing 
mutation), detected in a total of $48 \%$ of families. The predominant mutations in IL-10RB deficiency were c.139A>G (exon 2, p.K47E), c.477G>A (exon 4, p.W159X) and c.611G>A (exon 5, p.W204X), identified in $34 \%$ of families. Generally, most mutations were evenly located throughout the IL-10RA and IL-10RB genes, except for the hot spot mutations c.301C>T and c.537G>A in the IL-10RA gene. Large deletion mutations were reported in 21 families consisting of deletion containing exon $1(18,19)$, exon 1-3 $(20,21)$ and exon 2-4 (p.V23fsX31) (20) of the IL-10RA gene, and deletion in exon $2(22,23)$, exon 3 (g.34647434_34650412delinsCT) and duplication of exon 6 (24), deletion of exon 3-7 (25), exon 4 and part of exon 5 (g.11930_17413del) $(11,22)$, exon 4 and exon 5 (g.17030_22177del) $(21,26)$ of the $I L$ $10 R B$ gene.

These large deletion mutations were reported in 21 families and can be categorized into two subgroups: homozygous large deletions in 11 families [(2 in IL-10RA, 9 in IL-10RB) in which 4 cases were Asian, 5 European, and 2 unknowns] and compound heterozygous mutations (large deletion and missense mutations) in 9 families with IL-10RA deficiency [8 cases were Chinese and one unknown] and one family with IL-10RB (European). There was no difference in the clinical progression of these 21 families and other patients. One study reported 6 cases with more than 2 variants in IL-10RA (27). Of 10 variants identified in these cases, 3 were novel missense variants and were not reported in other publications: c.314T>A (p.1105N), c.670A>G (p.I224V), and c.884C>T (p.P295L). The clinical significance of these variants is unknown.

\subsection{Clinical findings}

The most common first presentation was chronic diarrhea reported by $45.7 \%$, bloody diarrhea by $18.1 \%$, and colitis by $15.7 \%$ of patients (Figure 4). Of 249 patients with available presenting diagnosis, 234 (94\%) were diagnosed with IBD and 8 (3.2\%) with food allergy (Figure S1). All patients reported gastrointestinal involvement with 243 cases having the diagnosis of colitis [49.8\% (121 of 243)], Crohn's disease [32.5\% (79 of 243)], or UIBD [17.7\% (43 of 243)]. Bloody diarrhea and chronic diarrhea were diagnosed in 80 and 84 patients, respectively. In addition to IBD which was reported in 100\% of patients, six patients also presented with other types of inflammatory/autoimmune disorders, including diabetes insipidus (2 cases), autoimmune hepatitis, immune thrombocytopenia (ITP), Behcet's disease, and autoimmune enteropathy each occurring in one case. Oral complications were present in $37.4 \%$ (64 of 171) patients, mostly in the form of ulcers $74.6 \%$ (49 of 64 patients). Ninety-one percentage of the patients (225 out of 245) had perianal manifestations including fistulae [58.7\% (101 of 172)], abscesses [60.8\% (102 of 176)], fissures [18.9\% (31 of 164)], and skin tags [17.7\% (29 of 164)]. The other complications commonly associated with IBD were malnutrition and failure to thrive (FTT) detected in more than half of the patients [58.1\% (90 of 155 with evaluable data)].

Other extra-intestinal features in order of frequency were: dermatologic manifestations [47.7\% [(93 of 195), mostly consisting of folliculitis and eczematous lesions], rheumatologic manifestations [13\% [(19 of 146), mostly arthritis/arthralgia], hematologic manifestations [6.5\% [(9 of 139), mainly anemia], renal disorders [6.9\% (9 of 130)], hepatobiliary disorders [3.9\% (5 of 128)], allergies [2.3\% (3 of 128)], endocrinopathies [3.7\% (5 of 134)], neurologic disorders [2.4\% (3 of 127)], and cardiovascular diseases 
[1.6\% (2 of 128)]. Hepatosplenomegaly and lymphadenopathy were observed in $5.5 \%$ and $5.4 \%$ of the patients, respectively. B cell lymphoma and a case of juvenile myelomonocytic leukemia were demonstrated in $12.1 \%$ of patients (18 of 149) (Table 3 ). Respiratory tract infections were reported in $22.8 \%$ of patients, including pneumonia $(11.5 \%)$, sinusitis $(2.4 \%)$, bronchitis $(1.6 \%)$, ear, nose, throat involvement (8.5\%). Septicemia, candidiasis, and meningitis occurred in $16.3 \%, 9.5 \%$, and $1.6 \%$ of patients, respectively. 
Table 3

- Clinical findings of patients with IL-10/IL-10R deficiency

\begin{tabular}{|c|c|c|c|c|c|c|c|}
\hline Parameters & Total & IL-10 & IL-10R & $\begin{array}{l}P \text { - } \\
\text { value }\end{array}$ & $\begin{array}{l}\text { IL- } \\
\text { 10RA }\end{array}$ & $\begin{array}{l}\text { IL- } \\
\text { 10RB }\end{array}$ & $\begin{array}{l}P \text { - } \\
\text { value }\end{array}$ \\
\hline \multicolumn{8}{|l|}{ Gastrointestinal involvements } \\
\hline $\begin{array}{l}\text { Gastrointestinal disorders, } n \text {, } \\
(\%)(n=284)\end{array}$ & $\begin{array}{l}284 \\
(100)\end{array}$ & $\begin{array}{l}18 \\
(100)\end{array}$ & $\begin{array}{l}266 \\
(100)\end{array}$ & - & $\begin{array}{l}188 \\
(100)\end{array}$ & $\begin{array}{l}69 \\
(100)\end{array}$ & - \\
\hline $\begin{array}{l}\text { Non-GI autoimmunity, } n,(\%) \\
(n=128)\end{array}$ & $4(3.1)$ & 0 & $4(3.3)$ & 1.000 & $4(4.5)$ & 0 & 0.575 \\
\hline Crohn's disease, $n,(\%)(n=243)$ & $\begin{array}{l}79 \\
(32.5)\end{array}$ & $9(50)$ & $\begin{array}{l}70 \\
(31.1)\end{array}$ & 0.100 & $\begin{array}{l}44 \\
(29.7)\end{array}$ & $\begin{array}{l}20 \\
(29.4)\end{array}$ & 0.962 \\
\hline Colitis, n, (\%) (n=243) & $\begin{array}{l}121 \\
(49.8)\end{array}$ & $\begin{array}{l}6 \\
(33.3)\end{array}$ & $\begin{array}{l}115 \\
(51.1)\end{array}$ & 0.147 & $71(48)$ & $\begin{array}{l}43 \\
(63.2)\end{array}$ & 0.037 \\
\hline UIBD, n, (\%) (n=243) & $\begin{array}{l}43 \\
(17.7)\end{array}$ & $\begin{array}{l}3 \\
(16.7)\end{array}$ & $\begin{array}{l}40 \\
(17.8)\end{array}$ & 0.905 & $\begin{array}{l}33 \\
(22.3)\end{array}$ & $\begin{array}{l}5 \\
(7.4)\end{array}$ & 0.007 \\
\hline $\begin{array}{l}\text { Perianal manifestations, } n,(\%) \\
(n=245)\end{array}$ & $\begin{array}{l}225 \\
(91.8)\end{array}$ & $\begin{array}{l}13 \\
(81.3)\end{array}$ & $\begin{array}{l}212 \\
(92.6)\end{array}$ & 0.131 & $\begin{array}{l}154 \\
(90.6)\end{array}$ & $\begin{array}{l}55 \\
(98.2)\end{array}$ & 0.078 \\
\hline Fissures, $n,(\%)(n=164)$ & $\begin{array}{l}31 \\
(18.9)\end{array}$ & 0 & $\begin{array}{l}31 \\
(19.1)\end{array}$ & 1.000 & $\begin{array}{l}27 \\
(22.1)\end{array}$ & $\begin{array}{l}4 \\
(10.3)\end{array}$ & 0.102 \\
\hline Fistulae, $n, \%(n=172)$ & $\begin{array}{l}101 \\
(58.7)\end{array}$ & $\begin{array}{l}2 \\
(100)\end{array}$ & $\begin{array}{l}99 \\
(58.2)\end{array}$ & 0.512 & $\begin{array}{l}81 \\
(63.3)\end{array}$ & $\begin{array}{l}17 \\
(41.5)\end{array}$ & 0.014 \\
\hline Abscesses, $n, \%(n=176)$ & $\begin{array}{l}102 \\
(58)\end{array}$ & 0 & $\begin{array}{l}102 \\
(59)\end{array}$ & 0.073 & $\begin{array}{l}74 \\
(58.7)\end{array}$ & $\begin{array}{l}28 \\
(60.9)\end{array}$ & 0.800 \\
\hline Failure to thrive, $n, \%(n=155)$ & $\begin{array}{l}90 \\
(58.1)\end{array}$ & 0 & $90(60)$ & 0.012 & $\begin{array}{l}66 \\
(64.7)\end{array}$ & $\begin{array}{l}20 \\
(47.6)\end{array}$ & 0.057 \\
\hline $\begin{array}{l}\text { Oral complications, } n, \% \\
(n=171)\end{array}$ & $\begin{array}{l}64 \\
(37.4)\end{array}$ & $1(20)$ & $63(38)$ & 0.652 & $\begin{array}{l}51 \\
(39.8)\end{array}$ & $\begin{array}{l}10 \\
(27.8)\end{array}$ & 0.186 \\
\hline $\begin{array}{l}\text { Hepatobiliary disorders, } n, \% \\
(n=128)\end{array}$ & $5(3.9)$ & 0 & $5(4.1)$ & 1.000 & $3(3.4)$ & $\begin{array}{l}2 \\
(5.7)\end{array}$ & 0.624 \\
\hline \multicolumn{8}{|l|}{ Infectious involvements } \\
\hline $\begin{array}{l}\text { Respiratory infections, } n, \% \\
(n=133)\end{array}$ & $\begin{array}{l}28 \\
(21.1)\end{array}$ & 0 & $\begin{array}{l}28 \\
(21.9)\end{array}$ & 0.584 & $\begin{array}{l}19 \\
(20.9)\end{array}$ & $\begin{array}{l}8 \\
(22.9)\end{array}$ & 0.808 \\
\hline Septicemia, n, \% (n=129) & $\begin{array}{l}21 \\
(16.3)\end{array}$ & 0 & $\begin{array}{l}21 \\
(16.9)\end{array}$ & 0.591 & $\begin{array}{l}17 \\
(19.3)\end{array}$ & $\begin{array}{l}4 \\
(11.4)\end{array}$ & 0.294 \\
\hline Candidiasis, $n, \%(n=126)$ & $\begin{array}{l}12 \\
(9.5)\end{array}$ & 0 & $\begin{array}{l}12 \\
(9.9)\end{array}$ & 1.000 & $\begin{array}{l}9 \\
(10.2)\end{array}$ & $\begin{array}{l}3 \\
(9.4)\end{array}$ & 1.000 \\
\hline Meningitis, $n(\%)(n=128)$ & $2(1.6)$ & 0 & $2(1.6)$ & 1.000 & 0 & $\begin{array}{l}2 \\
(5.7)\end{array}$ & 0.081 \\
\hline
\end{tabular}




\begin{tabular}{|c|c|c|c|c|c|c|c|}
\hline Parameters & Total & IL-10 & IL-10R & $\begin{array}{l}P- \\
\text { value }\end{array}$ & $\begin{array}{l}\text { IL- } \\
\text { 10RA }\end{array}$ & $\begin{array}{l}\text { IL- } \\
\text { 10RB }\end{array}$ & $\begin{array}{l}P \text { - } \\
\text { value }\end{array}$ \\
\hline Bronchiectasis, n (\%) (n=130) & $2(1.5)$ & 0 & $2(1.6)$ & 1.000 & - & $\begin{array}{l}2 \\
(5.6)\end{array}$ & 0.083 \\
\hline \multicolumn{8}{|l|}{ Lymphoproliferative involvements } \\
\hline Malignancy, $n, \%(n=149)$ & $\begin{array}{l}18 \\
(12.1)\end{array}$ & 0 & $\begin{array}{l}18 \\
(12.5)\end{array}$ & 1.000 & $5(5.3)$ & $\begin{array}{l}13 \\
(26.5)\end{array}$ & 0.001 \\
\hline $\begin{array}{l}\text { Hepatosplenomegaly, } n, \% \\
(n=128)\end{array}$ & $7(5.5)$ & 0 & $7(2.6)$ & 1.000 & $1(1.1)$ & $\begin{array}{l}6 \\
(17.1)\end{array}$ & 0.002 \\
\hline $\begin{array}{l}\text { Lymphadenopathy, } n, \% \\
(n=129)\end{array}$ & $7(5.4)$ & 0 & $7(5.6)$ & 1.000 & $1(1.1)$ & $\begin{array}{l}6 \\
(16.7)\end{array}$ & 0.003 \\
\hline \multicolumn{8}{|l|}{ Other involvements } \\
\hline $\begin{array}{l}\text { Dermatologic manifestations, } \\
n, \%(n=200)\end{array}$ & $\begin{array}{l}97 \\
(48.5)\end{array}$ & 0 & $\begin{array}{l}97 \\
(50.5)\end{array}$ & 0.007 & $\begin{array}{l}66 \\
(48.2)\end{array}$ & $\begin{array}{l}29 \\
(54.7)\end{array}$ & 0.419 \\
\hline $\begin{array}{l}\text { Rheumatologic disorders, } n, \% \\
(n=146)\end{array}$ & $19(13)$ & 0 & $\begin{array}{l}19 \\
(13.5)\end{array}$ & 1.000 & $5(5.6)$ & $\begin{array}{l}13 \\
(26.5)\end{array}$ & $<0.001$ \\
\hline Renal disorders, $n, \%(n=130)$ & $9(6.9)$ & 0 & $9(7.2)$ & 1.000 & $3(3.4)$ & $\begin{array}{l}6 \\
(16.7)\end{array}$ & 0.018 \\
\hline $\begin{array}{l}\text { Hematologic disorders, } n, \% \\
(n=139)\end{array}$ & $9(6.5)$ & 0 & $9(6.7)$ & 1.000 & $6(6.7)$ & $3(7)$ & 1.000 \\
\hline Endocrinopathy, $n, \%(n=134)$ & $5(3.7)$ & 0 & $5(3.9)$ & 1.000 & $4(4.5)$ & $\begin{array}{l}1 \\
(2.6)\end{array}$ & 1.000 \\
\hline $\begin{array}{l}\text { Neurologic disorders, } n(\%) \\
(n=127)\end{array}$ & $3(2.4)$ & 0 & $3(2.5)$ & 1.000 & $3(3.4)$ & 0 & 0.558 \\
\hline Allergy, $n(\%)(n=128)$ & $3(2.3)$ & 0 & $3(2.4)$ & 1.000 & $3(3.4)$ & 0 & 0.559 \\
\hline $\begin{array}{l}\text { Cardiovascular diseases, } n(\%) \\
(n=128)\end{array}$ & $2(1.6)$ & 0 & $2(1.6)$ & 1.000 & $1(1.1)$ & $\begin{array}{l}1 \\
(2.9)\end{array}$ & 0.493 \\
\hline \multicolumn{8}{|c|}{ n; number, GI; Gastrointestinal, UIBD; Undetermined inflammatory bowel disease } \\
\hline \multicolumn{8}{|c|}{${ }^{*} p$-value is statistically significant $<0.05$} \\
\hline
\end{tabular}

In patients with IL-10R deficiency the frequency of dermatologic manifestations (50.5\%) and failure to thrive $(60 \%)$ was high, while these complications were absent in IL-10-deficient patients (Table 3$)$. Proven enteropathy was present in 100\% [Crohn's disease (29.7\%), colitis (48\%), UIBD (22.3\%)] of IL-10RAdeficient and 100\% [Crohn's disease (29.4\%), colitis (63.2\%), UIBD (7.4\%)] of IL-10RB-deficient patients. While the frequency of colitis was higher in IL-10RB deficiency $(p=0.037)$, UIBD was more often reported in IL-10RA-deficient cases ( $\mathbf{p}=\mathbf{0 . 0 0 7}$ ). Other significant differences between IL-10RA and IL-10RB were observed in the incidence of fistulae which occurred in $63.3 \%$ of patients with IL-10RA deficiency vs. $41.5 \%$ of patients with IL-10RB deficiency $(p=0.014)$, rheumatologic presentations [IL-10RA deficiency 
(5.6\%) vs. IL-10RB deficiency (26.5\%); $\mathrm{p}<0.001$ ], renal disorders [IL-10RA deficiency (3.4\%), vs. IL-10RB deficiency (16.7\%); $p=0.018$ ]. Moreover, lymphoproliferation including lymphadenopathy $(1.1 \%$ vs. $16.7 \%$; $p=0.003)$, hepatosplenomegaly $(1.1 \%$ vs. $17.1 \% ; p=0.002)$, and particularly malignancy $(5.3 \%$ vs. $26.5 \%$; $\mathrm{p}=0.001$ ) were more frequent in IL-10RB deficiency compared to IL-10RA deficiency.

\subsection{Immunologic evaluations}

In the majority of patients with available immunologic data, most basic immunologic parameters were found to be within normal ranges. Those included $89.6 \%$ with normal CD3+ counts (60 of 67 ), $88.1 \%$ with normal CD4+ counts (59 of 67), 88.2\% with normal CD8+ T cell counts (60 of 68 ), $88.1 \%$ with normal CD19+ B cell counts (59 of 67), and $97.5 \%$ with normal CD16/56+ NK cell counts (39 of 40). Immunoglobulin levels [IgG (80.5\%), IgA (72.5\%), IgM (84.6\%), and IgE (87.1\%)] were mostly normal, while elevated $\lg G(17.1 \%), \lg A(27.5 \%), \lg M(15.4 \%)$, and $\lg E(12.9 \%)$ values were reported in a small subset of patients. STAT3-phosphorylation and IL-10 expression were reported in a few articles. Ninety-three percent of all patients with available data (26 of 28) showed a lack of STAT 3-phosphorylation [21 (91.3\%) patients with IL-10RA deficiency vs. $5(100 \%)$ of cases with IL-10RB deficiency]. All studied IL10RA-deficient patients had normal $(41.7 \%)$ or elevated (58.3\%) serum levels of IL-10. Basic immunologic profiles were not significantly different between the patients with IL-10 deficiency vs. patients with IL-10R deficiency, nor in comparison of patients with IL-10RA deficiency vs. patients with IL-10RB deficiency.

\subsection{Therapeutic approach}

Most patients (65.6\%) were treated with corticosteroids, while only a few $(2$ of $81,2.5 \%)$ responded appropriately. Immunosuppressive drugs were tried in $87 \%$ of patients with only $3.7 \%$ (4 of 108 cases) responding. Immunosuppressive drugs consisted of monoclonal antibodies [infliximab, adalimumab, rituximab, and anakinra, in the order of prevalence] were used in 74 cases, azathioprine in 60 cases, and thalidomide in 48 cases. Intravenous immunoglobulin (IVIG) was given as a suppressive treatment to 21 patients. Another treatment tried in 66 patients was 5-aminosalicylic acid (5-ASA). One hundred and seven patients (61.5\%, of 174 with available data) underwent surgery preferably colostomy and colectomy. Steroid therapy and surgical procedures were performed more often in patients with IL-10RA deficiency compared to patients with IL-10RB deficiency ( $p<0.001$ and $p=0.002$, respectively, Table S1).

Fifty-six out of 187 patients with available data underwent HSCT from different sources [cord (55.9\%), bone marrow (26.5\%), and peripheral blood (17.6\%)] at median (IQR) age of $2.0(1.0-6.0)$ years with $83.9 \%$ (47 of 56) achieving remission. In patients with available data, neutrophil and thrombocyte engraftment occurred at median (IQR) times of 21.0 (13.0-23.2) days and 26.0 (19.0-29.0) days after transplantation, respectively. If mentioned, conditioning regimens were reduced intensity (10 cases) and myeloablative (4 cases).

Kaplan-Meier curves (Figure 5) do not indicate significant differences in the survival rate of patients with IL-10 and IL-10R deficiency ( $p=0.233)$, nor in those of patients with IL-10RA and IL-10RB deficiency $(p=0.122)$. However, the ten-year survival rate of patients with IL-10 deficiency $(100 \%)$ was higher than patients with IL-10R deficiency (70\%). Overall, $83.9 \%$ (47 of 56 ) of all patients who underwent HSCT 
survived (compared to $74.8 \%$ of patients who did not undergo HSCT, $p=0.190$ ). Complete recovery was achieved in $83.3 \%, 78.4 \%$, and $93.3 \%$ of IL-10R-deficient, IL-10RA-deficient, and IL-10RB-deficient patients following transplantation ( $p=0.209, p=0.428, p=0.095$, respectively, Figure $\mathbf{S} 2$ ). Furthermore, we compared the survival of patients who underwent HSCT and found a higher but not statistically significant threeyear survival rate in patients with IL-10RB deficiency (90\%) compared to patients with IL-10RA deficiency $(80 \%, p=0.268$, Figure S3).

\section{Discussion}

In this study, we systematically reviewed the clinical, immunological, and genetic aspects of 284 patients with IL-10/IL-10R deficiency. Most patients were of East Asia origin and presented with features that were initially diagnosed as IBD within their first months of life. Ninety-three percent of patients (of the 263 with available data) were less than 2 years old at age of onset and were subsequently categorized as VEOIBD which is similar to a recent publication revealing higher prevalence and earlier onset of IL-10/IL-10R among Asian populations (28). Taken together, mitigating VEOIBD should raise the suspicion for IL-10/IL10R deficiency and prompt genetic evaluation. Considering the autosomal recessive inheritance (16), the overall estimated rate of consanguinity and positive family history of IBD in the patients reported here was less than currently estimated (29). However, in agreement with other inborn errors of immunity, genetic evaluation should be considered in families with a member previously diagnosed with early-onset IBD, especially in the presence of consanguinity. Of note, IL-10 and IL-10RB deficiency had a notably higher rate of consanguinity than IL-10RA deficiency, respectively.

Loss-of-function mutations in IL-10, IL-10RA, or IL-10RB genes are the cause of severe phenotypes of infantile IBD. In this review of 265 patients with a confirmed mutation in IL-10, IL-10RA or IL 10-RB genes, 90 unique mutations at the DNA level were identified. The c.301C>T (p.R101W) and c.537G>A (p.T179T) with a total of $48 \%$ of cases were the most prevalent variants in IL-10RA. Because of the distinct clinical presentations observed in siblings with the same mutation (10) and based on previous cohort studies (20), there is no clear genotype-phenotype correlation, suggesting that the phenotypic manifestations are dependent on intrinsic or extrinsic factors that remain unknown. The complex and diverse phenotypes and genetic heterogeneity of IL-10/IL-10R deficiencies reflect, to some extent, the variable clinical phenotypes of IBDs. Monogenic or polygenic mechanisms combined with environmental factors may affect susceptibility, severity and onset of the disease (27). Girardelli et al. reported 6 patients with a digenic inheritance that had double heterozygous mutations in IL-10RA and IL-10RB (27). In these patients, two heterozygous variants of two genes may additively disturb the IL-10 receptor alpha and beta chain hetero-tetrameric structure. Sometimes, these variants are located in two or more genes with different signaling pathways that have a cumulative effect (30). This may explain how reported heterozygous mutations in IL-10RA and IL-10RB are associated with a pathologic phenotype (31-34). Due to the multimeric structure of the IL-10 receptor, the dominant-negative effect of these heterozygous variants may be the underlying reason (31). Additional analyses will be required to determine the exact effects of these digenic variants on immune cell function (34). 
As has been suggested by simple-context-based observations $(5,10)$, our comprehensive review confirmed that the prevalence of various clinical manifestations, especially extra-intestinal involvements, was higher in IL-10R deficiency compared to IL-10 deficiency and in IL-10RB deficiency than IL-10RA deficiency. In line with this observation, a higher ten-year survival rate was present in patients with IL-10 deficiency vs. patients with IL-10R deficiency (Figure 5A). This difference may be explained by the increased number of IL-10RB ligands. Unlike IL-10RA, IL-10RB binds not only to IL-10, but also connects with other members of the IL-10 cytokine family including IL-22, IL-26, IL-28, and IL-29 contributing to the attenuation of inflammatory responses (35). Therefore, more severe clinical phenotypes observed in patients with IL-10RB deficiency may be related to increased cytokine activity in affected organs (36-41). Manifestations with significantly higher prevalence in IL-10RB deficiency included fistula formation, rheumatologic and renal disorders, along with hepatosplenomegaly and malignancy. Patients with IL-10R deficiency presented with a higher occurrence of dermatologic manifestations and failure to thrive. Furthermore, the impact of IL-10RA and IL-10RB deficiency differed in the incidence of UIBD and colitis. Of note, the importance of IL-10RB in regulating host susceptibility to severe acute respiratory syndrome coronavirus 2 (SARS-CoV-2) and suppression of cytokine storm has been suggested (42). Thus, aside from a more severe phenotype, an increased probability of SARS-CoV-2 disease is anticipated among patients carrying IL-10RB mutations.

No significant immunologic abnormalities were apparent, except for an elevated level of IL-10 in cases with IL-10RA deficiency which could be explained by defective binding/consumption of IL-10 in patients lacking its receptor. Despite the central role of IL-10 in B cell differentiation into IgA secreting plasma cells which are involved in mucosal immunity $(43,44)$, elevated IgA levels were observed in $27.4 \%$ of the patients, all carrying IL-10R mutations. This could be explained by $T$ cell-independent IgA synthesis during chronic IBD inflammation $(45,46)$. STAT 3-phosphorylation, a key mediator in the IL-10 pathway (2), was absent in $93 \%$ of IL-10/IL-10R-deficient patients with available data. However, the observed immunological results indicate that IL-10/IL-10R deficiency minimally affects the adaptive immune systems. Although steroids and immunosuppressive drugs were the main therapeutic strategies, the patients' clinical responses were unimpressive. HSCT seems to be the most effective therapeutic approach with a high probability of remission in IL-10/IL-10R-deficient patients. However, detailed data related to transplantation were missing in almost half of the cases which made recommendations regarding HSCT less accurate.

\section{Conclusions}

In summary, our review recommends that IL-10/IL-10R deficiency should be considered as a cause of VEOIBD when occurring within the first months of life in patient's resistant to conventional therapies. No clear genotype-phenotype correlation could be identified. Distinct clinical manifestations reported in IL10R and IL-10RB-deficient patients are attributable to cytokines other than IL- 10 sharing IL-10RB and contributing to inflammation attenuation. HSCT is currently the most effective therapeutic approach and improved the survival rate of all patients with IL-10/IL-10R deficiencies. However, the statistical insignificance of HSCT compared to conservative treatment, probably results from the missing data of a 
considerable number of patients and the upcoming studies should target this aspect of IL-10/IL-10R deficiency.

\section{Statements And Declaration}

Funding: This work was supported by Alborz University of Medical Sciences (Grant number 4039).

Competing Interests: The authors have no relevant financial or non-financial interests to disclose.

Author Contributions: Gholamreza azizi contributed to the study conception and design. Material preparation, data collection and analysis were performed by Niusha Sharifinejad, Majid Zaki-Dizaji, Roya Sepahvandi and Farimah Fayyaz. The first draft of the manuscript was written by Niusha Sharifinejad and Majid Zaki-Dizaji. The other authors commented on previous versions of the manuscript. All authors read and approved the final manuscript.

Data Availability: The datasets generated during and/or analysed during the current study are available from the corresponding author on reasonable request.

Ethics approval: Not applicable.

Consent to participate/publish: Not applicable.

Acknowledgments: None.

\section{References}

1. Standiford TJ, Deng JC. INTERLEUKINS | IL-10. In: Laurent GJ, Shapiro SD, editors. Encyclopedia of Respiratory Medicine. Oxford: Academic Press; 2006. p. 373-7.

2. Iyer SS, Cheng G. Role of interleukin 10 transcriptional regulation in inflammation and autoimmune disease. Crit Rev Immunol. 2012;32(1):23-63.

3. Walter MR. The molecular basis of IL-10 function: from receptor structure to the onset of signaling. Current topics in microbiology and immunology. 2014;380:191-212.

4. Glocker EO, Kotlarz D, Klein C, Shah N, Grimbacher B. IL-10 and IL-10 receptor defects in humans. Ann N Y Acad Sci. 2011;1246:102-7.

5. Engelhardt KR, Grimbacher B. IL-10 in humans: lessons from the gut, IL-10/IL-10 receptor deficiencies, and IL-10 polymorphisms. Current topics in microbiology and immunology. 2014;380:118.

6. Tangye SG, Al-Herz W, Bousfiha A, Chatila T, Cunningham-Rundles C, Etzioni A, et al. Human Inborn Errors of Immunity: 2019 Update on the Classification from the International Union of Immunological Societies Expert Committee. Journal of clinical immunology. 2020;40(1):24-64. 
7. Rosen MJ, Dhawan A, Saeed SA. Inflammatory Bowel Disease in Children and Adolescents. JAMA Pediatr. 2015;169(11):1053-60.

8. Uhlig HH, Schwerd T, Koletzko S, Shah N, Kammermeier J, Elkadri A, et al. The diagnostic approach to monogenic very early onset inflammatory bowel disease. Gastroenterology. 2014;147(5):9901007.e3.

9. Thurgate LE, Lemberg DA, Day AS, Leach ST. An Overview of Inflammatory Bowel Disease Unclassified in Children. Inflammatory intestinal diseases. 2019;4(3):97-103.

10. Kotlarz D, Beier R, Murugan D, Diestelhorst J, Jensen O, Boztug K, et al. Loss of interleukin-10 signaling and infantile inflammatory bowel disease: implications for diagnosis and therapy. Gastroenterology. 2012;143(2):347-55.

11. Neven B, Mamessier E, Bruneau J, Kaltenbach S, Kotlarz D, Suarez F, et al. A Mendelian predisposition to B-cell lymphoma caused by IL-10R deficiency. Blood. 2013;122(23):3713-22.

12. Shah N, Kammermeier J, Elawad M, Glocker EO. Interleukin-10 and interleukin-10-receptor defects in inflammatory bowel disease. Current allergy and asthma reports. 2012;12(5):373-9.

13. Uhlig HH, Charbit-Henrion F, Kotlarz D, Shouval DS, Schwerd T, Strisciuglio C, et al. Clinical Genomics for the Diagnosis of Monogenic forms of Inflammatory Bowel Disease: A Position Paper from The Paediatric IBD Porto Group of ESPGHAN. Journal of pediatric gastroenterology and nutrition. 2020;Publish Ahead of Print.

14. Engelhardt KR, Shah N, Faizura-Yeop I, Kocacik Uygun DF, Frede N, Muise AM, et al. Clinical outcome in IL-10- and IL-10 receptor-deficient patients with or without hematopoietic stem cell transplantation. The Journal of allergy and clinical immunology. 2013;131(3):825-30.

15. Shouval DS, Biswas A, Kang YH, Griffith AE, Konnikova L, Mascanfroni ID, et al. Interleukin $1 \beta$ Mediates Intestinal Inflammation in Mice and Patients With Interleukin 10 Receptor Deficiency. Gastroenterology. 2016;151(6):1100-4.

16. Krawiec P, Pawłowska-Kamieniak A, Pac-Kożuchowska E. Interleukin 10 and interleukin 10 receptor in paediatric inflammatory bowel disease: from bench to bedside lesson. Journal of Inflammation. 2021;18(1):13.

17. Sharifinejad N, Zaki-Dizaji M, Tebyanian S, Zainaldain H, Jamee M, Rizvi FS, et al. Clinical, immunological, and genetic features in 938 patients with autoimmune polyendocrinopathy candidiasis ectodermal dystrophy (APECED): a systematic review. Expert Rev Clin Immunol. 2021:111.

18. Tang Z, Zhang P, Ji M, Yin C, Zhao R, Huang Z, et al. Characterization of novel and large fragment deletions in exon 1 of the IL10RA gene in Chinese children with very early onset inflammatory bowel diseases. BMC gastroenterology. 2021;21(1):167.

19. Dong F, Xiao F, Ge T, Li X, Xu W, Wu S, et al. Case Report: A Novel Compound Heterozygous Mutation in IL-10RA in a Chinese Child With Very Early-Onset Inflammatory Bowel Disease. Front Pediatr. 2021;9:678390. 
20. Engelhardt KR, Shah N, Faizura-Yeop I, Uygun DFK, Frede N, Muise AM, et al. Clinical outcome in IL10-and IL-10 receptor-deficient patients with or without hematopoietic stem cell transplantation. Journal of Allergy and Clinical Immunology. 2013;131(3):825-+.

21. Shouval DS, Konnikova L, Griffith AE, Wall SM, Biswas A, Werner L, et al. Enhanced TH17 Responses in Patients with IL10 Receptor Deficiency and Infantile-onset IBD. Inflammatory bowel diseases. 2017;23(11):1950-61.

22. Pigneur B, Escher J, Elawad M, Lima R, Buderus S, Kierkus J, et al. Phenotypic characterization of very early-onset IBD due to mutations in the IL10, IL10 receptor alpha or beta gene: a survey of the Genius Working Group. Inflammatory bowel diseases. 2013;19(13):2820-8.

23. Uygun DFK, Uygun V, Daloglu H, Ozturkmen S, Karasu G, Reisli I, et al. Hematopoietic Stem Cell Transplantation From Unrelated Donors in 2 Cases of Interleukin-10 Receptor Deficiency: Is Surgery Not a Requirement? Journal of Pediatric Hematology Oncology. 2019;41(1):64-6.

24. Charbit-Henrion F, Bègue B, Sierra A, Hanein S, Stolzenberg MC, Li Z, et al. Copy number variations and founder effect underlying complete IL-10R $\beta$ deficiency in Portuguese kindreds. PloS one. 2018;13(10):e0205826.

25. Rahmani F, Rayzan E, Rahmani MR, Shahkarami S, Zoghi S, Rezaei A, et al. Clinical and Mutation Description of the First Iranian Cohort of Infantile Inflammatory Bowel Disease: The Iranian Primary Immunodeficiency Registry (IPIDR). Immunological investigations. 2020:1-15.

26. Shouval DS, Biswas A, Goettel JA, McCann K, Conaway E, Redhu NS, et al. Interleukin-10 receptor signaling in innate immune cells regulates mucosal immune tolerance and anti-inflammatory macrophage function. Immunity. 2014;40(5):706-19.

27. Girardelli M, Basaldella F, Della Paolera S, Vuch J, Tommasini A, Martelossi S, et al. Genetic profile of patients with early onset inflammatory bowel disease. Gene. 2018;645:18-29.

28. Huang JG, Aw MM. Pediatric Inflammatory Bowel Disease in Asia: Epidemiology and natural history. Pediatrics and neonatology. 2020;61(3):263-71.

29. Almana Y, Mohammed R. Current concepts in pediatric inflammatory bowel disease; IL 10/IL10R colitis as a model disease. Int J Pediatr Adolesc Med. 2019;6(1):1-5.

30. Girardelli M, Vuch J, Tommasini A, Crovella S, Bianco AM. Novel missense mutation in the NOD2 gene in a patient with early onset ulcerative colitis: causal or chance association? International journal of molecular sciences. 2014;15(3):3834-41.

31. Nemati S, Teimourian S, Tabrizi M, Najafi M, Dara N, Imanzadeh F, et al. Very early onset inflammatory bowel disease: Investigation of the IL-10 signaling pathway in Iranian children. European journal of medical genetics. 2017;60(12):643-9.

32. Xiao Y, Wang XQ, Yu Y, Guo Y, Xu X, Gong L, et al. Comprehensive mutation screening for 10 genes in Chinese patients suffering very early onset inflammatory bowel disease. World journal of gastroenterology. 2016;22(24):5578-88.

33. Oh SH, Baek J, Kim KM, Lee EJ, Jung Y, Lee YJ, et al. Is Whole Exome Sequencing Clinically Practical in the Management of Pediatric Crohn's Disease? Gut and liver. 2015;9(6):767-75. 
34. Kelsen JR, Dawany N, Moran CJ, Petersen BS, Sarmady M, Sasson A, et al. Exome sequencing analysis reveals variants in primary immunodeficiency genes in patients with very early onset inflammatory bowel disease. Gastroenterology. 2015;149(6):1415-24.

35. Shouval DS, Ouahed J, Biswas A, Goettel JA, Horwitz BH, Klein C, et al. Interleukin 10 receptor signaling: master regulator of intestinal mucosal homeostasis in mice and humans. Adv Immunol. 2014;122:177-210.

36. Broggi A, Tan Y, Granucci F, Zanoni I. IFN- $\lambda$ suppresses intestinal inflammation by non-translational regulation of neutrophil function. Nat Immunol. 2017;18(10):1084-93.

37. Lu T, Liu Y, Yu S, Yin C, Li P, Ye J, et al. Increased frequency of circulating Th22 cells in patients with B-cell non-Hodgkin's lymphoma. Oncotarget. 2016;7(35).

38. Pott J, Stockinger S. Type I and III Interferon in the Gut: Tight Balance between Host Protection and Immunopathology. Frontiers in immunology. 2017;8(258).

39. Shen W, Hixon JA, McLean MH, Li WQ, Durum SK. IL-22-Expressing Murine Lymphocytes Display Plasticity and Pathogenicity in Reporter Mice. Frontiers in immunology. 2016;6(662).

40. Weidenbusch M, Song S, Iwakura T, Shi C, Rodler S, Kobold S, et al. IL-22 sustains epithelial integrity in progressive kidney remodeling and fibrosis. Physiol Rep. 2018;6(16):e13817.

41. Wongthida P, Diaz RM, Galivo F, Kottke T, Thompson J, Pulido J, et al. Type III IFN interleukin-28 mediates the antitumor efficacy of oncolytic virus VSV in immune-competent mouse models of cancer. Cancer Res. 2010;70(11):4539-49.

42. Voloudakis G, Hoffman G, Venkatesh S, Lee KM, Dobrindt K, Vicari JM, et al. IL10RB as a key regulator of COVID-19 host susceptibility and severity. medRxiv. 2021.

43. Brière F, Bridon JM, Chevet $D$, Souillet $G$, Bienvenu F, Guret $C$, et al. Interleukin 10 induces $B$ lymphocytes from IgA-deficient patients to secrete $\lg A$. The Journal of clinical investigation. 1994;94(1):97-104.

44. Sandy NS, Marega LF, Bechara GD, Riccetto AGL, Bonfim C, Vilela M, et al. Elevated IgA and IL-10 levels in very-early-onset inflammatory bowel disease secondary to IL-10 receptor deficiency. Rev Paul Pediatr. 2021;40:e2020434.

45. Rehnberg J, Symreng A, Ludvigsson JF, Emilsson L. Inflammatory Bowel Disease Is More Common in Patients with IgA Nephropathy and Predicts Progression of ESKD: A Swedish Population-Based Cohort Study. J Am Soc Nephrol. 2021;32(2):411-23.

46. Tezuka H, Ohteki T. Regulation of IgA Production by Intestinal Dendritic Cells and Related Cells. Frontiers in immunology. 2019;10(1891).

\section{Figures}



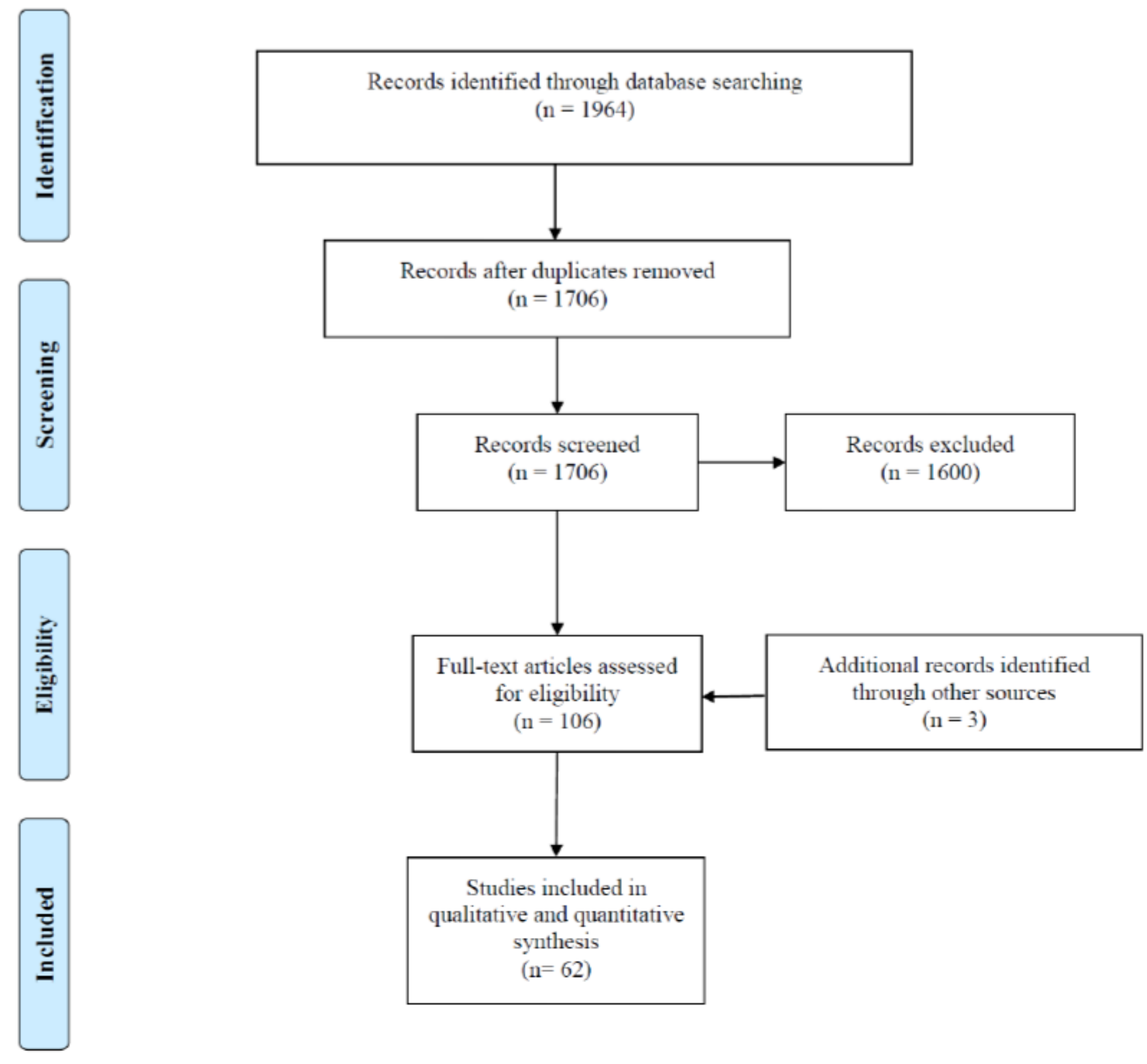

Figure 1

Study selection flow chart. 


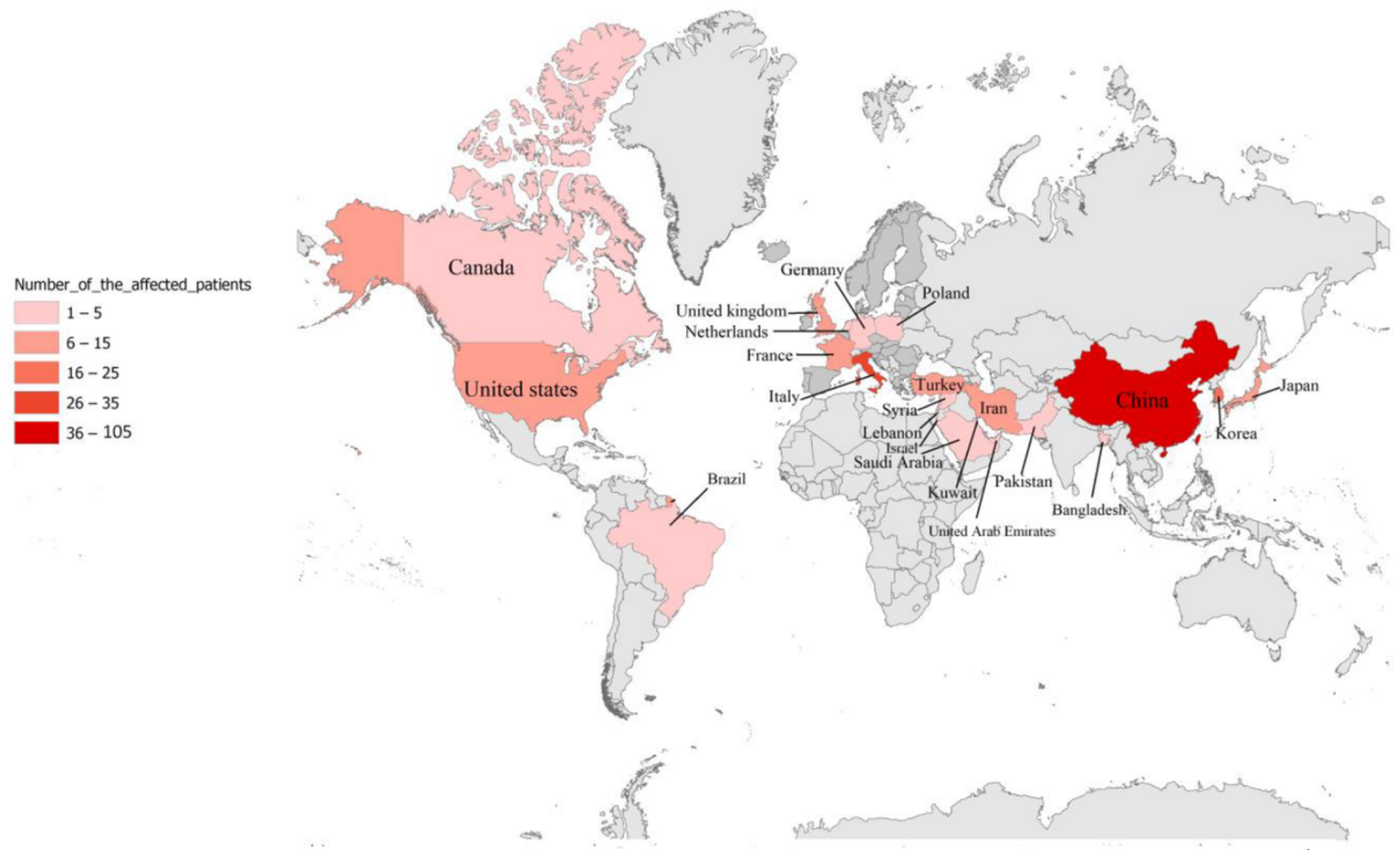

Figure 2

Worldwide distribution of patients with IL-10 and/or IL-10R deficiency. Most reported cases originated from China, Italy, and South Korea. 


\section{IL10 Gene}

UTR

Exon 1

Exon 2

Exon 3

Exon 4

Exon 5

UTR

\section{IL10RA Gene}

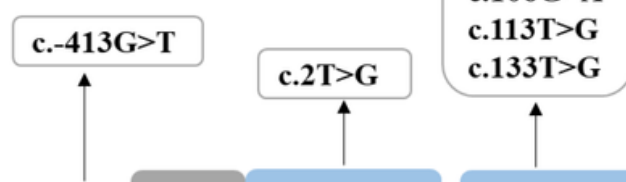

c. $191 \mathrm{~A}>\mathrm{G}$
$\mathrm{c} .205 \mathrm{~T}>\mathrm{C}$
$\mathrm{c} .251 \mathrm{C}>\mathrm{T}$
$\mathrm{c} .269 \mathrm{~T}>\mathrm{C}$
$\mathrm{c} .272 \mathrm{~A}>\mathrm{G}$
c. $299 \mathrm{~T}>\mathrm{G}$
c.436delC

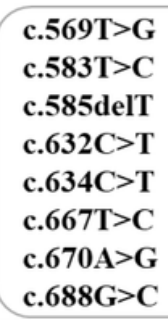

c.569T $>\mathrm{G}$ c.583T $>C$ c.585delT c. $632 \mathrm{C}>\mathrm{T}$ c. $634 \mathrm{C}>\mathrm{T}$ c. $667 \mathrm{~T}>\mathrm{C}$ c. $670 \mathrm{~A}>\mathrm{G}$ c. $688 \mathrm{G}>\mathrm{C}$

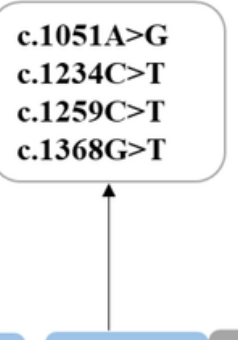

c.1259C $>$ T

c.1368G $>T$

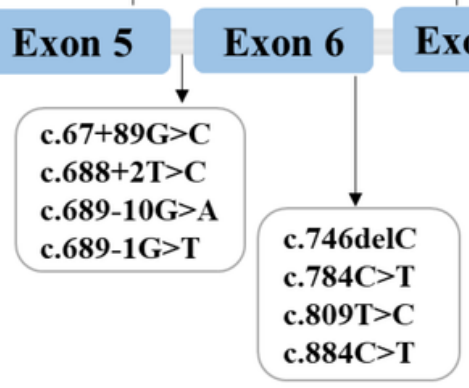

\section{IL10RB Gene}

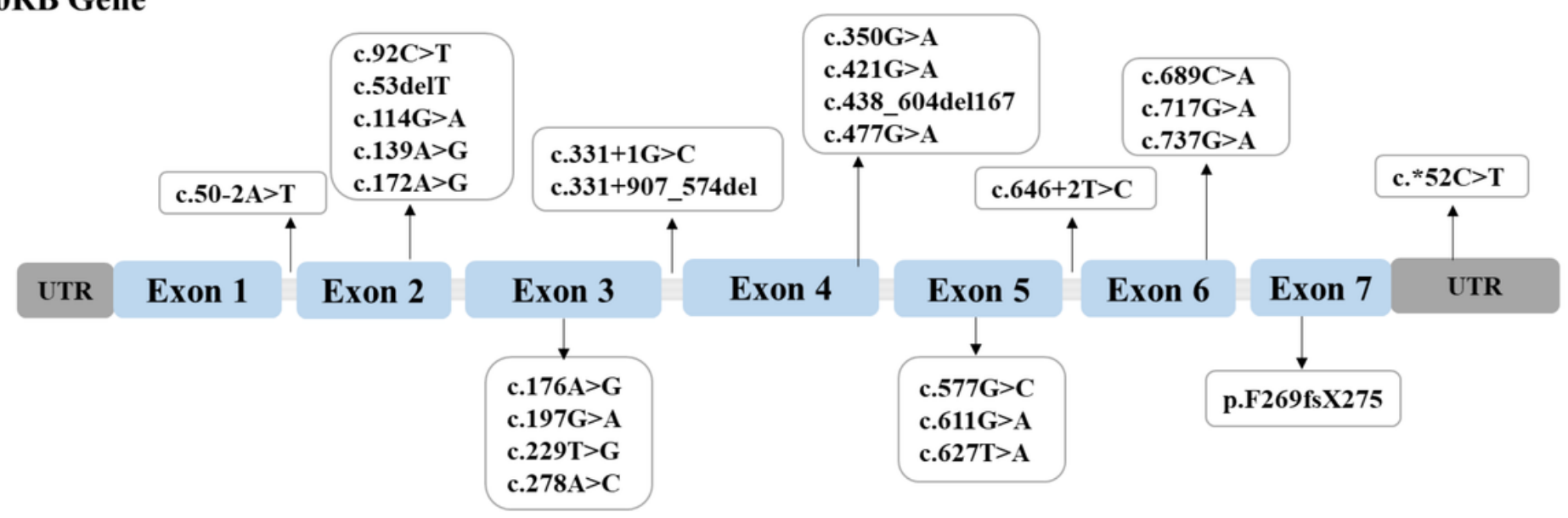

Figure 3

Graphical illustration of IL-10, IL-10RA and IL-10RB genes with locations of published genetic mutations in patients with IL-10 and IL-10 receptor deficiency. The eight reported large deletions in IL-10RA and IL10RB genes have been described in the text and not displayed in the figure. 


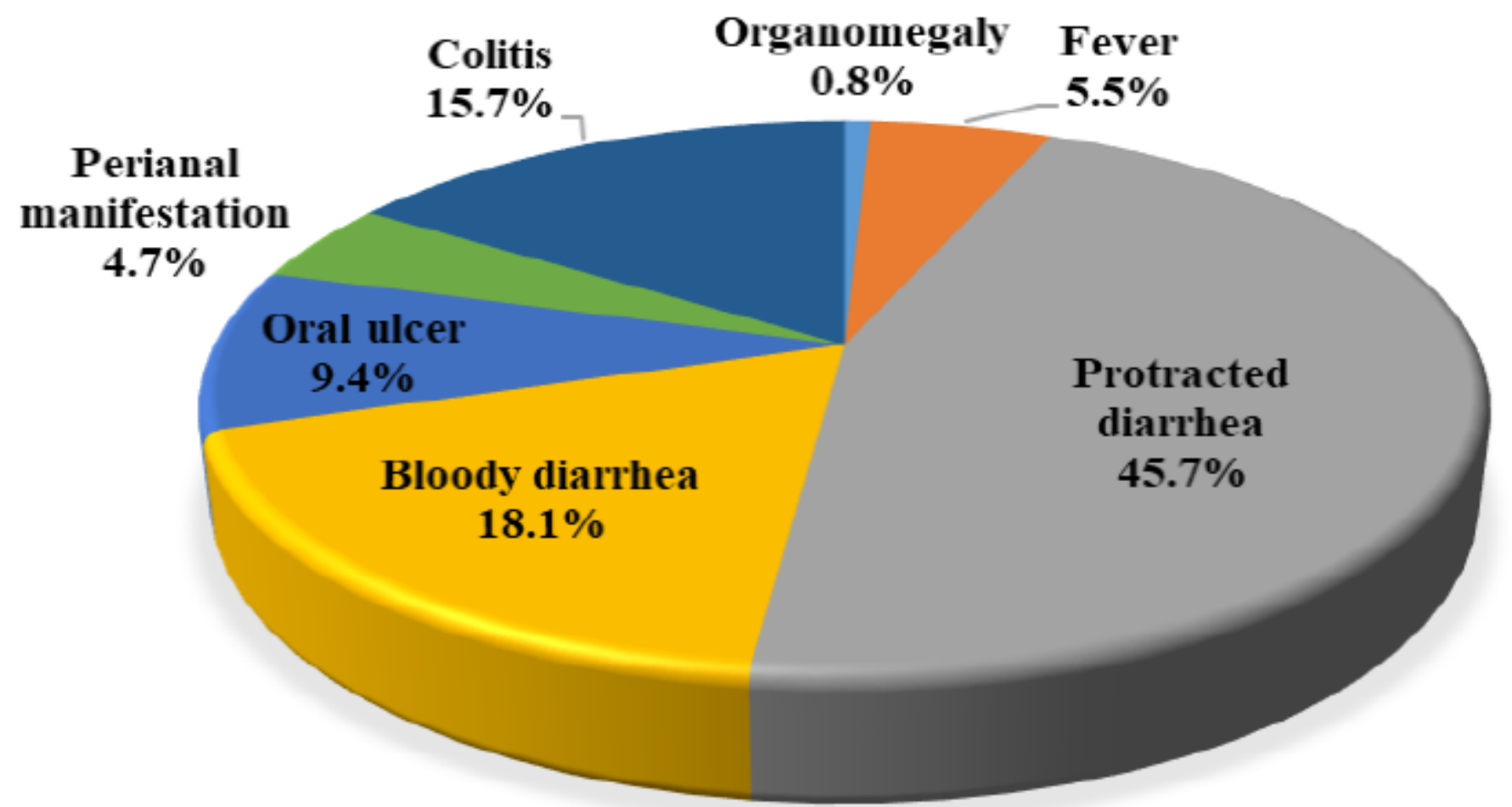

Figure 4

Initial presentations of patients $(n=127)$ with IL-10 and/or IL-10R deficiencies. 


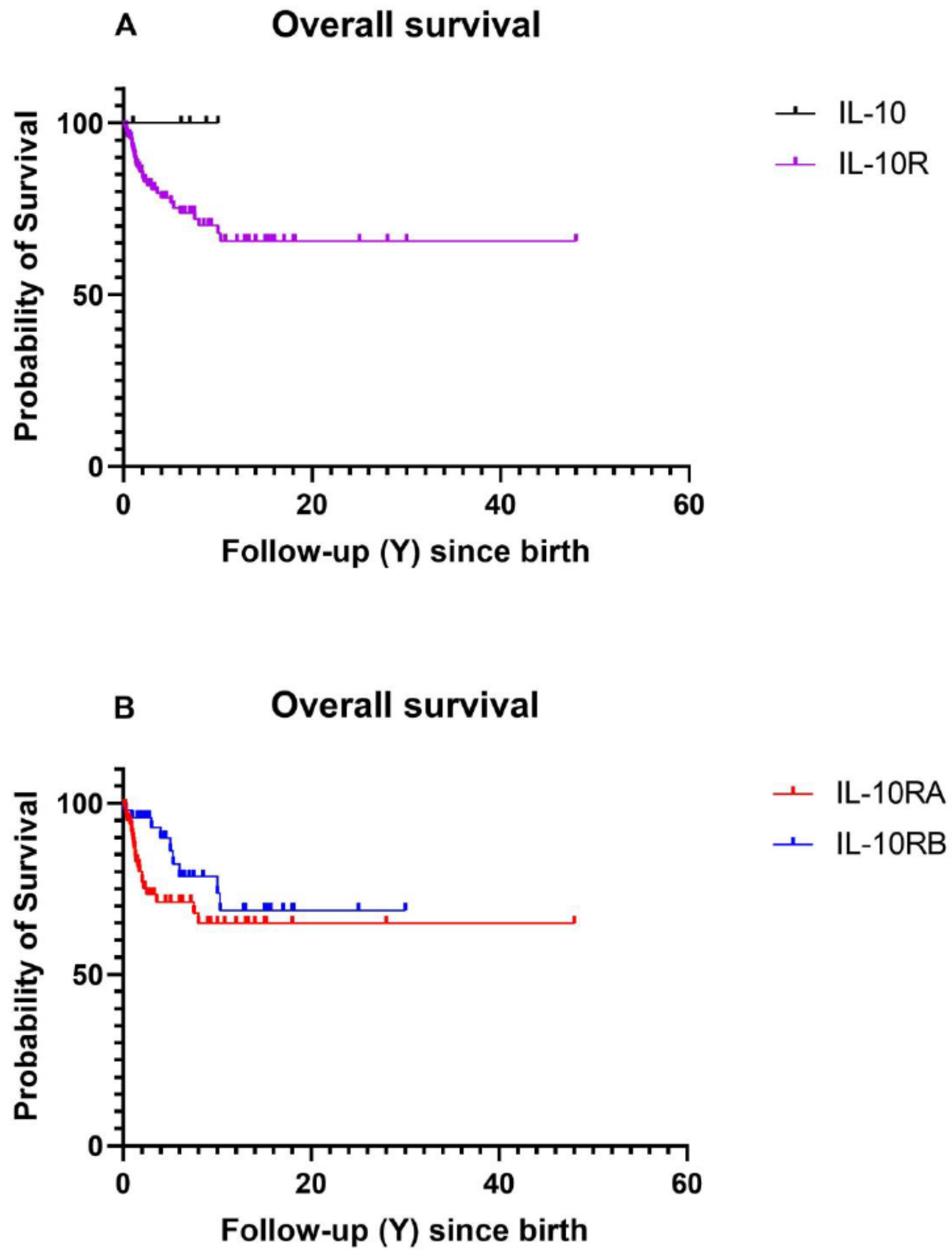

Figure 5

Kaplan-Meier survival curves of patients with IL-10 and IL-10R deficiencies (A) and patients with IL-10RA and IL-10RB deficiencies (B).

Supplementary Files 
This is a list of supplementary files associated with this preprint. Click to download.

- Supplementarymaterials.docx 